\title{
Assessing evidence of interventions addressing inequity among migrant populations: a two-stage systematic review
}

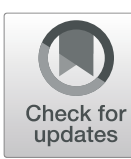

\author{
Jirawit Yadee ${ }^{1}$, Mukdarut Bangpan², Kednapa Thavorn ${ }^{3,4,5}$, Vivian Welch ${ }^{3,4,6}$, Peter Tugwell ${ }^{4,7,8,9}$ and \\ Nathorn Chaiyakunapruk ${ }^{10,11,12,13,14^{*}}$
}

\begin{abstract}
Background: Everyone has the right to achieve the standard of health and well-being. Migrants are considered as vulnerable populations due to the lack of access to health services and financial protection in health. Several interventions have been developed to improve migrant population health, but little is known about whether these interventions have considered the issue of equity as part of their outcome measurement.

Objective: To assess the evidence of health interventions in addressing inequity among migrants.

Methods: We adopted a two-stage searching approach to ensure the feasibility of this review. First, reviews of interventions for migrants were searched from five databases: PubMed, Cochrane, CINAHL, PsycINFO, and EMBASE until June 2017. Second, full articles included in the identified reviews were retrieved. Primary studies included in the identified reviews were then evaluated as to whether they met the following criteria: experimental studies which include equity aspects as part of their outcome measurement, based on equity attributes defined by PROGRESS-Plus factors (place of residence, race/ethnicity, occupation, gender, religion, education, socio-economic status, social capital, and others). We analysed the information extracted from the selected articles based on the PRISMA-Equity guidelines and the PROGRESSPlus factors.

Results: Forty-nine reviews involving 1145 primary studies met the first-stage inclusion criteria. After exclusion of 764 studies, the remaining 381 experimental studies were assessed. Thirteen out of 381 experimental studies (3.41\%) were found to include equity attributes as part of their outcome measurement. However, although some associations were found none of the included studies demonstrated the effect of the intervention on reducing inequity. All studies were conducted in high-income countries. The interventions included individual directed, community education and peer navigator-related interventions.
\end{abstract}

Conclusions: Current evidence reveals that there is a paucity of studies assessing equity attributes of health interventions developed for migrant populations. This indicates that equity has not been receiving attention in these studies of migrant populations. More attention to equity-focused outcome assessment is needed to help policy-makers to consider all relevant outcomes for sound decision making concerning migrants.

Keywords: Migrant, Health, Intervention, Equity

\footnotetext{
* Correspondence: nathorn.chaiyakunapruk@monash.edu

${ }^{10}$ School of Pharmacy, Monash University Malaysia, Jalan Lagoon Selatan,

46150 Bandar Sunway, Selangor, Malaysia

${ }^{11}$ Center of Pharmaceutical Outcomes Research (CPOR), Department of

Pharmacy Practice, Faculty of Pharmaceutical Sciences, Naresuan University,

Phitsanulok, Thailand

Full list of author information is available at the end of the article
}

(c) The Author(s). 2019 Open Access This article is distributed under the terms of the Creative Commons Attribution 4.0 International License (http://creativecommons.org/licenses/by/4.0/), which permits unrestricted use, distribution, and reproduction in any medium, provided you give appropriate credit to the original author(s) and the source, provide a link to the Creative Commons license, and indicate if changes were made. The Creative Commons Public Domain Dedication waiver (http://creativecommons.org/publicdomain/zero/1.0/) applies to the data made available in this article, unless otherwise stated. 


\section{Introduction}

Migrant populations often face health inequities which occur from inequalities in social and economic conditions [1] that impact on the risk of illness and the need for healthcare. In addition, migrants are often vulnerable due to lack of access to health services and financial protection in health [2]. According to the United Nations (UN) population division estimate, the number of international migrants has dramatically increased since 1960, rising from 77 million to almost 244 million in 2015, $3.3 \%$ of the world's population [3].

According to the World Health Organization (WHO) Constitution of 1948, the right to achieve the standard of health and well-being belongs to everyone including migrants and refugees [4]. In 2016, The 17 Sustainable Development Goals (SDGs) of the 2030 Agenda for Sustainable Development officially came into force. This agenda affirmed the need for monitoring to ensure that no one is left behind. To reduce inequalities, health policies should contribute to the achievement of SDG 3 on ensuring healthy lives and promoting well-being for all, SDG 5 on achieving gender equality, and SDG 10.7 on reducing inequalities by facilitating orderly, safe, and responsible migration and mobility of people, including through implementation of planned and well-managed migration policies. It is important for government and health systems to consider these aspects, to understand the barriers to health, and to then initiate interventions and approaches to improve the health of disadvantaged populations $[1,5]$.

A number of reviews have reported the effects of interventions aimed at improving health in migrant populations [6-14] with clinically relevant outcomes. However, little is known about whether these interventions have considered the issue of equity and used relevant equity-focused outcomes as part of their assessment. In addition, the inequity issue is crucial for the health system and is considered as a part of the global strategy. Health interventions should not only improve clinical outcomes but should also address the social disparity issue which is one of the SDGs. This review assesses whether health interventions reduce health inequities within migrant populations or consider equity as part of their measurement among the migrant populations. Findings from our study are expected to improve the understanding of current health intervention studies targeted at migrants. The review is particularly important since migrant populations are considered in the context of achieving target 10 of the global strategy on reducing inequities.

\section{Methods}

The review is reported according to the PRISMAEquity 2012 Statement [15] (Preferred Reporting Items for Systematic Review and Meta-Analysis with a focus on health Equity). We defined migrant populations as any people who moved across an international border away from their original place of residence, regardless of the voluntariness of movement [16]. To gather the evidence on equity attributes addressed in this review, we used the PROGRESS-Plus framework by members of the Campbell and Cochrane Equity Methods group [17].

\section{Literature search}

Since there have been a number of reviews on interventions developed to improve population health of migrant populations in the literature [6-14], we adopted a two-stage searching approach to ensure the feasibility of this review. First, a search was conducted in five databases (PubMed, Cochrane, CINAHL, PsycINFO, and EMBASE) from inception to June 2017. The main search terms for literature searches included "Migrant", "Health" and "Review". We adopted the search terms for "immigrant" and "systematic review". Further details on search terms used in literature search are shown in Table 1. A detailed example of a full electronic search is placed in Additional file 1: Table S1. There was no language restriction in this systematic review. We searched for reviews in this first stage and then retrieved the full articles of the studies included in each review to check whether they met the inclusion criteria. Second, we screened primary studies identified from each review which met the criteria from the first stage.

\section{Inclusion criteria}

Two stages of inclusion criteria were used. To be included in the first stage review, the review must meet the following inclusion criteria; [1] a review that reported the effect of health interventions [2] a review that included experimental studies comparing intervention(s) to standard/control group or before/after interventions [3] participants were migrants or immigrant, refugee, asylum seekers, or internally displaced persons. In the second stage, we included only primary studies with an experimental study design (randomized controlled trials or quasi-experimental studies) because these designs allow meaningful evaluation when equity is included as part of the outcome measurement of the health intervention. This is consistent with the goal of this review which is to determine whether evaluation of interventions have included equity attributes as part of their outcome measurement. The equity attributes of interest covered both social and economic risk factors using the PROGRESS-Plus categories (place of residence, race/ethnicity, occupation, gender, religion, education, socio-economic status, social capital, and others) as part of the outcome measurement. Further detail on the inclusion criteria of both stages is shown in Table 2. 
Table 1 Search terms

\begin{tabular}{ll}
\hline Search terms \\
\hline Literature review & "data synthesis" OR "evidence synthesis" OR metasynthesis OR meta-synthesis OR "narrative synthesis" OR "qualitative synthesis" OR \\
& "quantitative synthesis" OR "realist synthesis" OR "research synthesis" OR "synthesis of evidence" OR "thematic synthesis" OR \\
& metaanaly* OR meta-analy* OR "scoping stud*" OR meta-ethnograph* OR meta-epidemiological OR "systematic review" OR \\
"scoping review" OR "rapid review" & \\
Migrant & refugees OR refugee OR refugee camps OR camp OR refugee OR camps OR aliens OR alien OR emigrants OR emigrant OR \\
& foreigners OR foreigner OR immigrants OR immigrant OR migrant OR migrants OR asylum-seekers OR "internally displaced person" \\
Health & Health
\end{tabular}

* truncation operator represents zero or more terminal characters in a search term

\section{Article screening and data extraction}

One reviewer (JY) conducted the literature search, performed the screening and information extraction from the included studies. One reviewer (MB) verified the extracted data. Full texts of articles passing the second stage screening were retrieved, and their eligibility and quality assessed independently by two reviewers (NC and KT). Any discrepancy in the screening process was resolved through discussion. Two independent reviewers discussed the results. Extracted information included authors, year of study, study population, outcomes specified in the included studies, and findings related to PROGRESS-Plus factors as determinants of health equity.

\section{Quality assessment and data analysis}

Quality assessment for randomized controlled trials was performed using the Revised Cochrane risk of bias tool for randomized trials (RoB) version 2.0 [18]. Quality assessment for quasi-experimental studies was guided by Risk Of Bias In Non-randomized Studies of Interventions (ROBINS-I) [19]. Since there was no quantitative data similar enough to be pooled across studies, we could not perform quantitative synthesis. It was also not possible to use the GRADE (Grading of Recommendations Assessment, Development and Evaluation) framework for consideration of health equity as the overall effect estimates and uncertainty could not be estimated in our study. We narratively summarized all findings using a content analysis approach $[20,21]$.

\section{Results}

Study selection

We identified 2007 records, in which 49 reviews met the first-stage inclusion criteria. Of these, 1145 primary studies were screened to identify experimental studies which addressed health equity of migrants, using one or more PROGRESS-Plus factors. Based on the title and abstract screening, 120 and 644 were excluded because they were duplicates and non-experimental study designs, respectively. Out of the remaining 381 experimental studies, 324 did not include health equity attributes, leaving 57 studies for full-text retrieval. A total of 13 studies was included in this review after review at full text stage for eligibility. The flow of the included studies in this review is shown in Fig. 1.

\section{Study characteristics}

Table 3 shows the summary of results extracted from the included articles. Studies included in this review were conducted in the United States (US) $(N=11)$ [2232], Spain $(N=1)$ [33], and Norway $(N=1)$ [34]. The publication year ranged from 2000 to 2015 . We included eight randomized controlled studies (RCT) and five quasi-experimental studies.

Table 2 Inclusion criteria

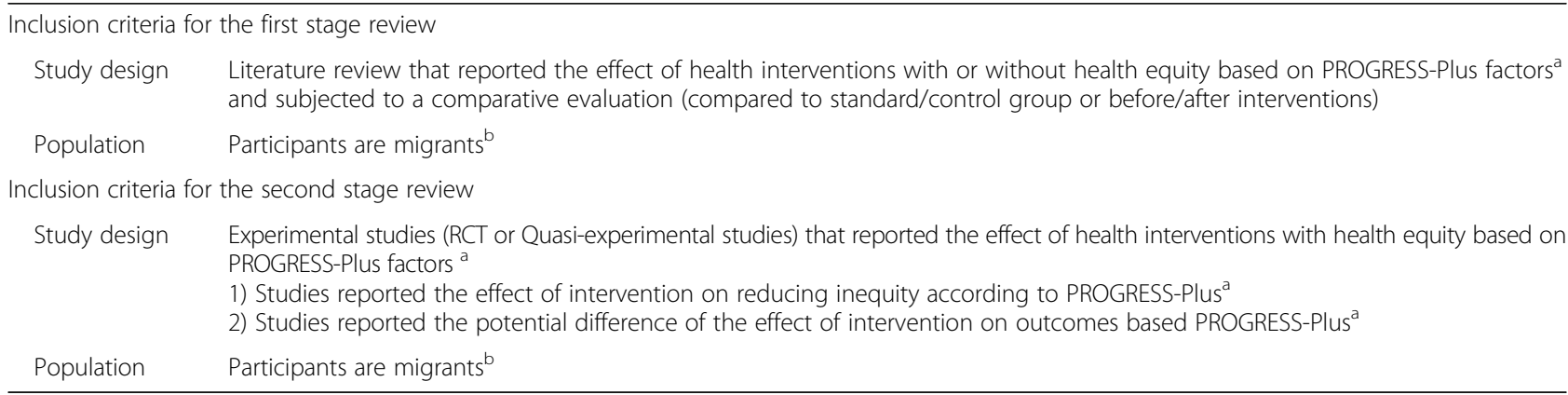

${ }^{a}$ Data on health equity: PROGRESS-Plus - Place of residence, Race/ethnicity/culture/language, Occupation, Gender/sex, Religion, Education, Socioeconomic status, Social capital and "Plus" to indicate other possible factors such as disease status or disability

$\mathrm{b}^{\mathrm{b}}$ Including other terms of migrants: immigrant, refugee, asylum seekers, and internally displaced person 


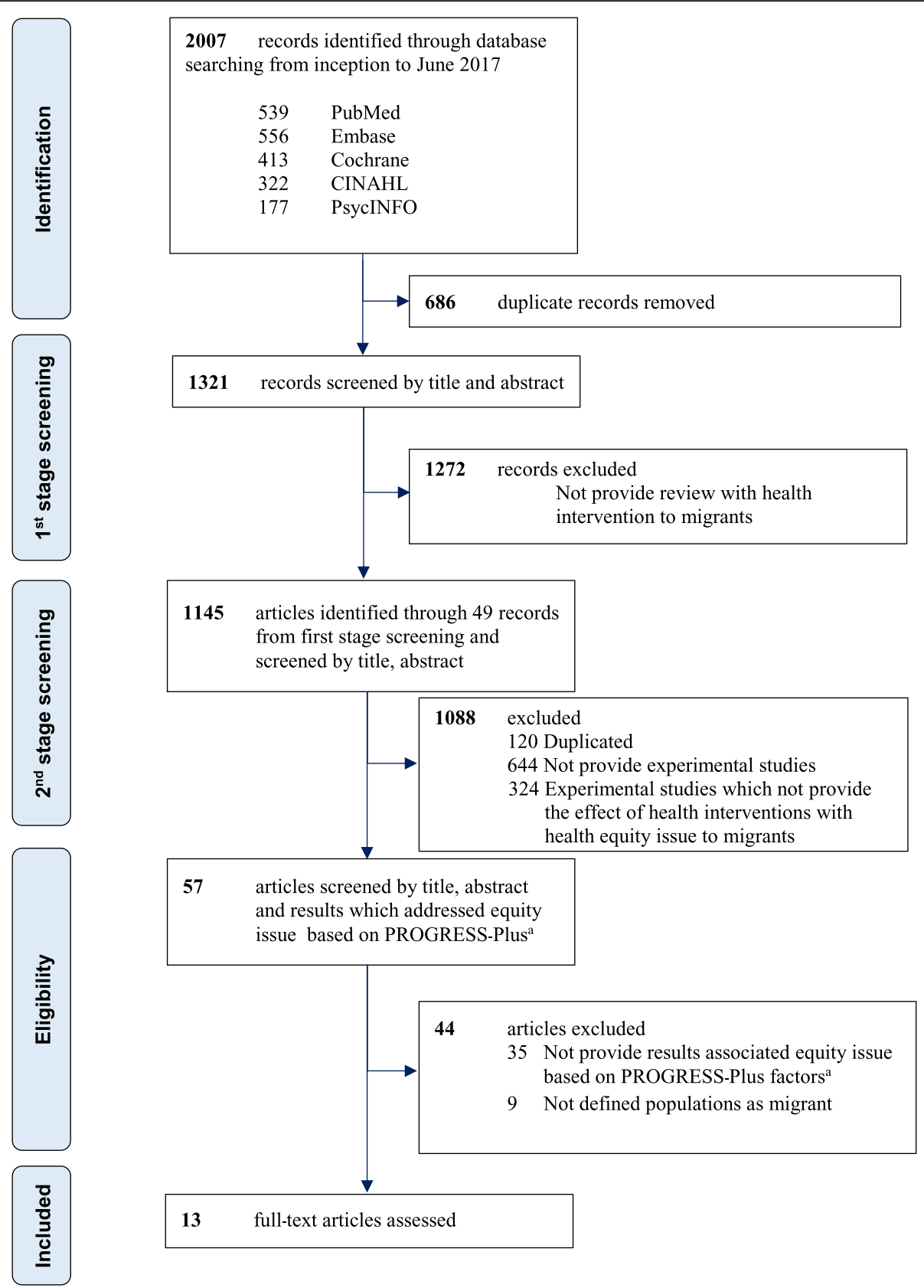

Fig. 1 PRISMA Flow Diagram of the literature search and selection process. 'Data on health equity: PROGRESS-Plus - Place of residence, Race/ethnicity/ culture/language, Occupation, Gender/sex, Religion, Education, Socioeconomic status, Social capital and "Plus" to indicate other possible factors such as disease status or disability

Following the study classification system used in the scoping review in migrant populations [6,35], these 13 studies were categorized as individual directed $(N=5)$ $[24,25,31,33,34]$, community education $(N=8)[22$, $23,26-29,32,34]$ and peer-navigator related intervention $(N=3)[26,30,32]$ (Fig. 2). The individual directed interventions aimed to provide the information on the benefits of a screening program or health intervention. Examples included the use of patient navigation by calling individuals prior to a screening colonoscopy procedure, mailing the instructions for bowel preparation after the scheduling call, and emphasizing the importance of the screening for the Latin migrants in the US [24]. Other individual directed interventions were the incorporation of nutritional behaviour change instruction into English-language curriculum for the Latin migrants in the US [25], the provision of an education session about the importance of diet and 


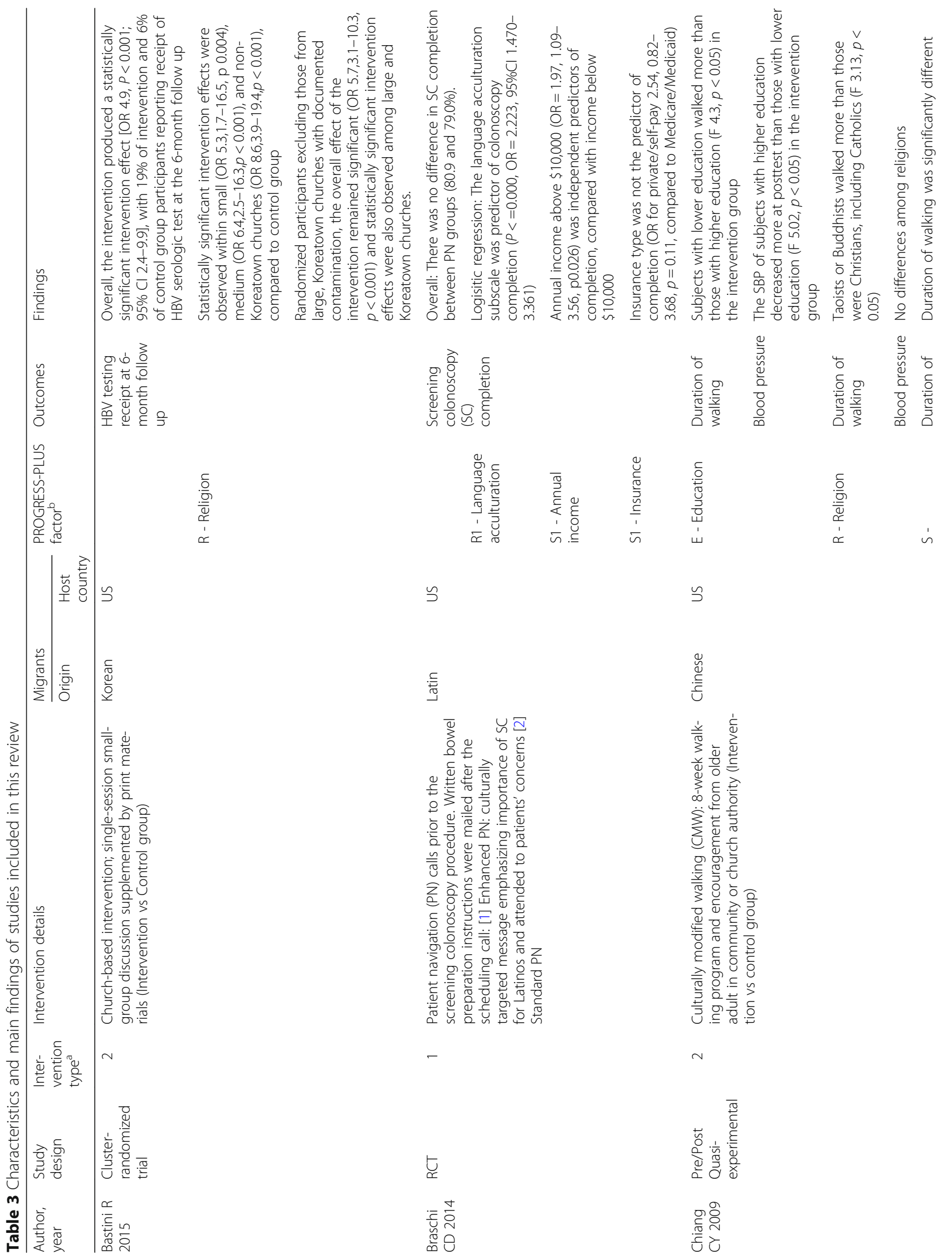




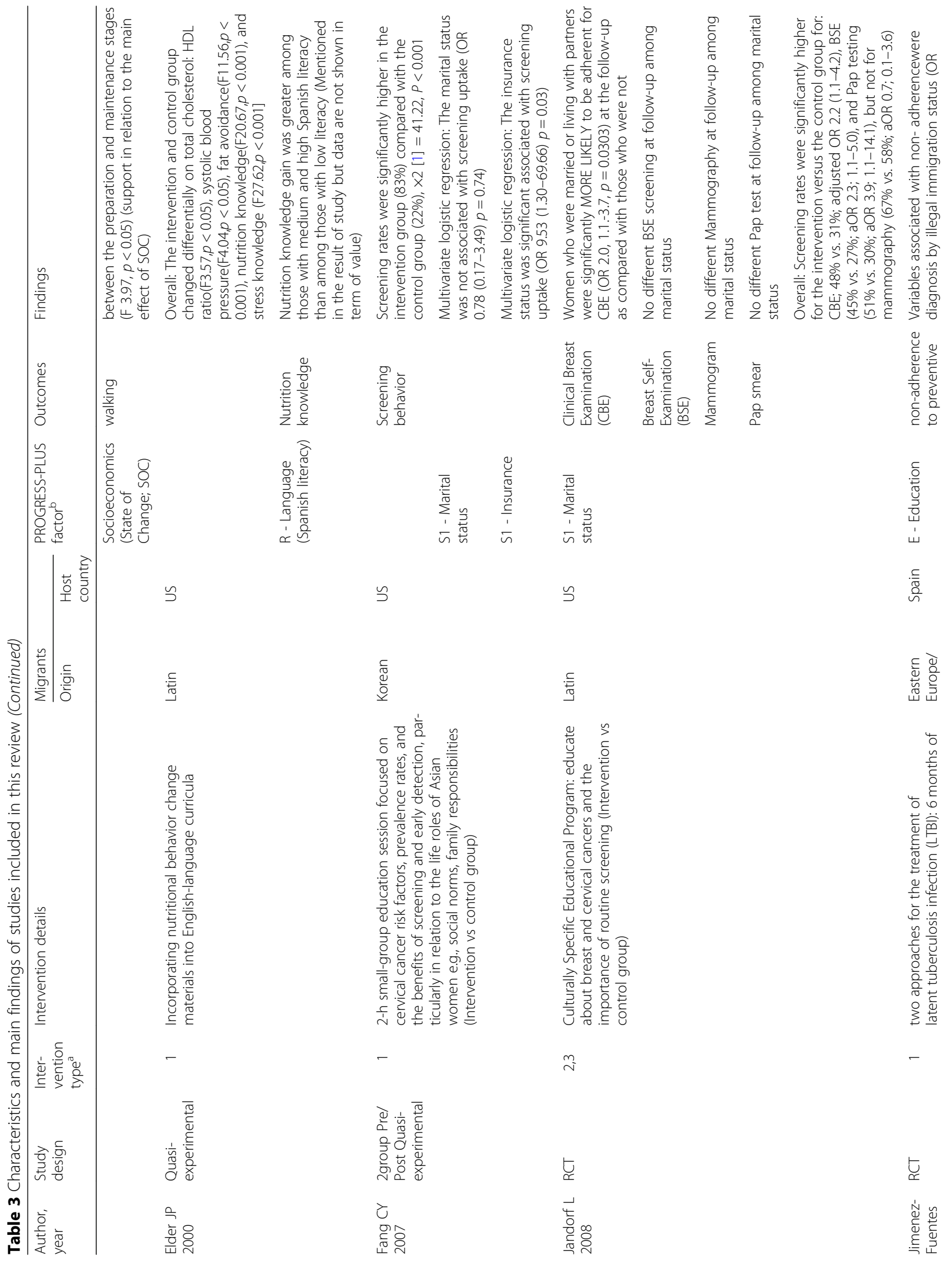




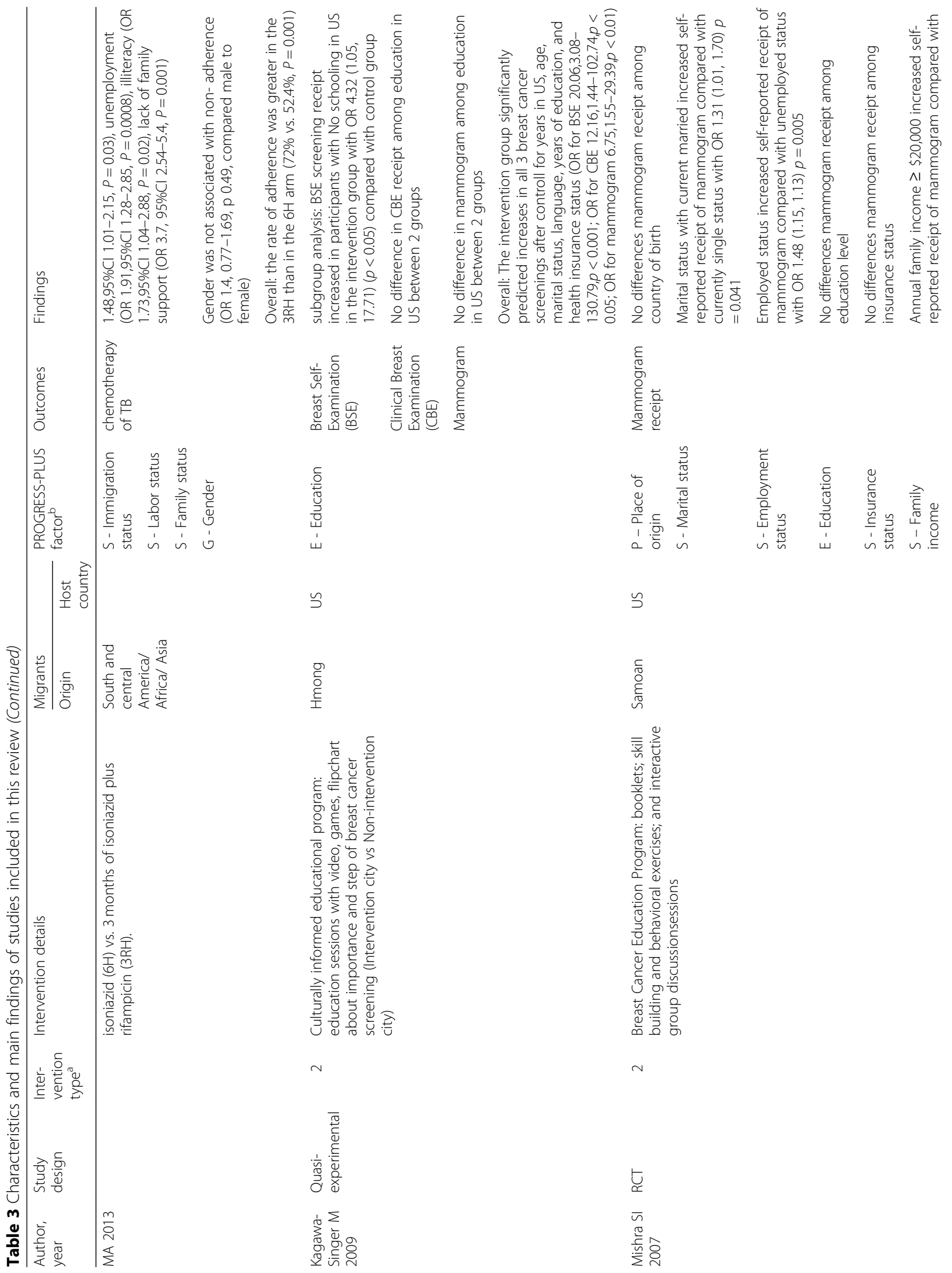




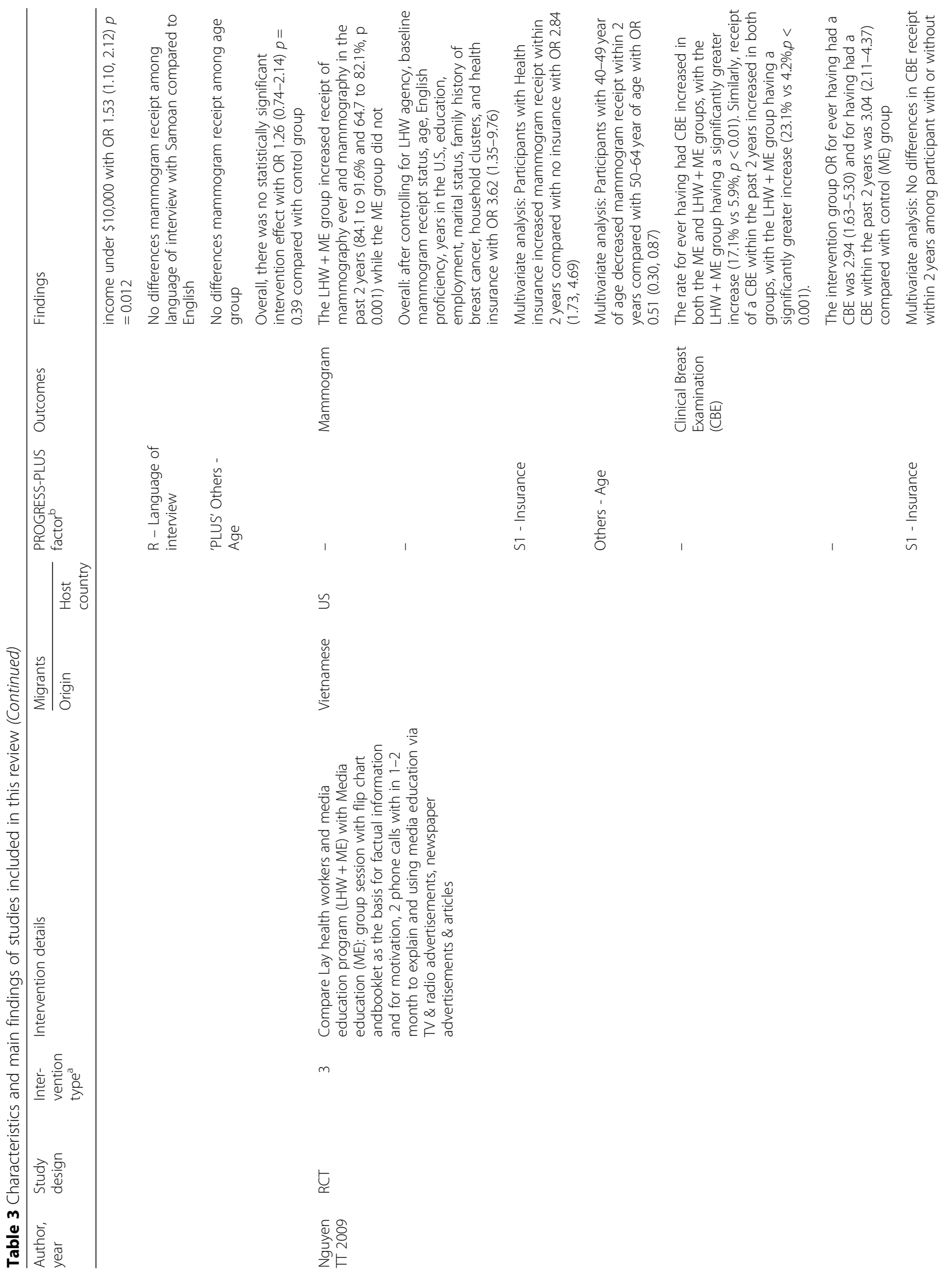




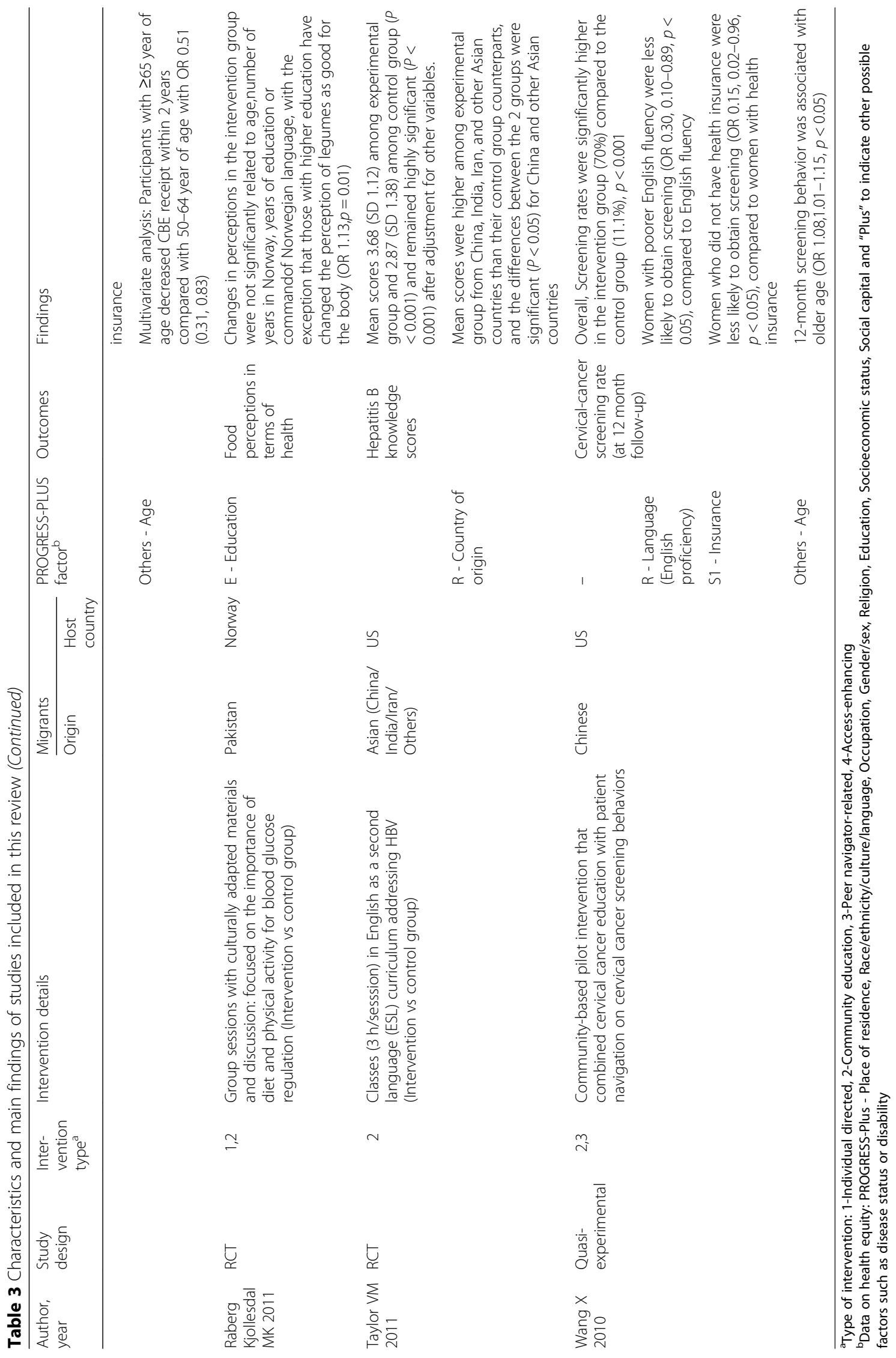




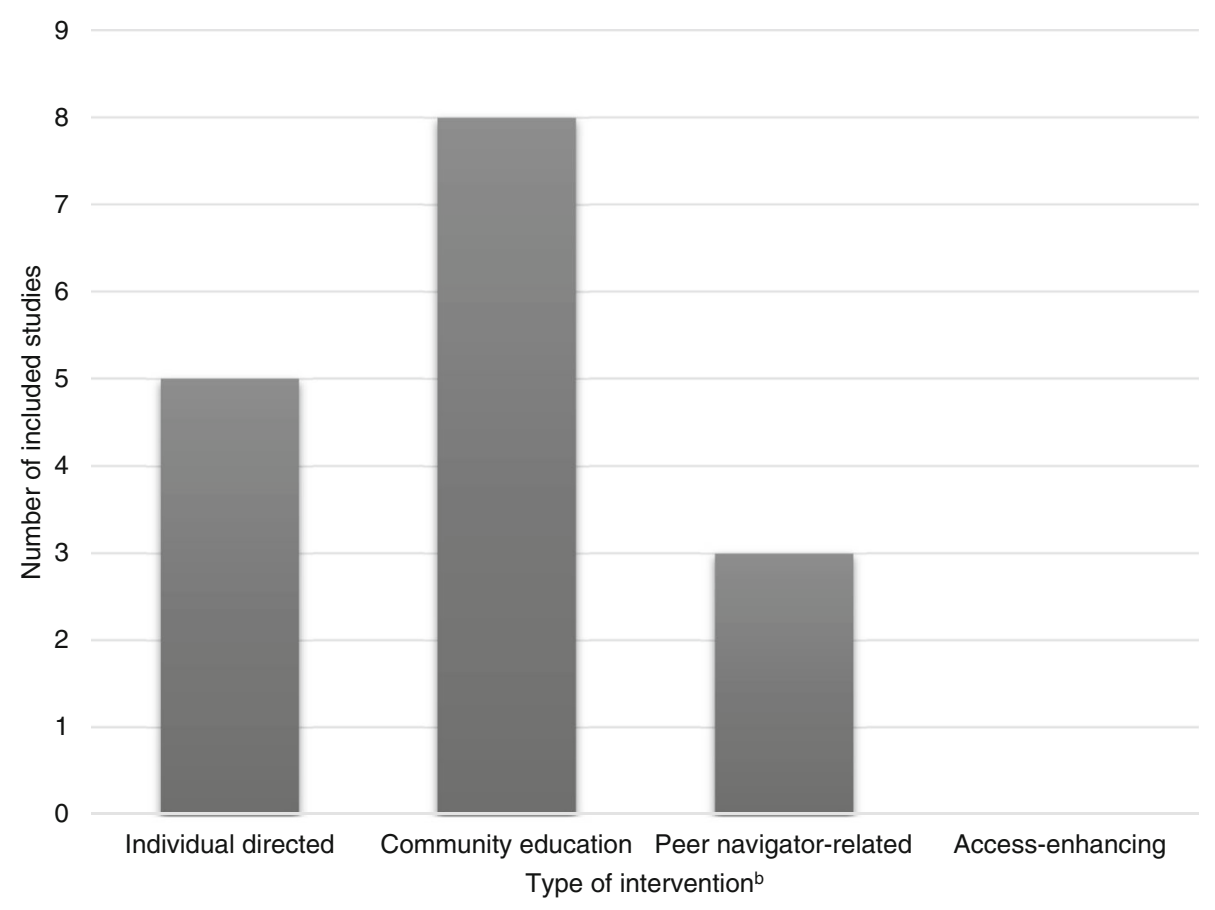

Fig. 2 Studies reported the type of intervention in this reviewa. ${ }^{2}$ Type of intervention- Individual directed intervention: to provide information to individual on benefits of screening or intervention; Community education: to provide the intervention through small group workshops or seminars usually conducted by healthcare professionals or trained staffs in the setting; Peer navigator-related intervention: the method by the peer leaders or lay health workers in the community to provide necessary support, follow-up, or referral to help migrants to receive intervention Access-enhancing: to promote screening by reducing financial or linguistic barriers that hamper access to screening services. ${ }^{b}$ Some studies reported more than one type

physical activity for blood glucose regulation for Pakistan migrants in Norway [34] or cervical cancer risk factors, prevalence rates, and the benefits of screening and early detection for Korean people [31] in the US, and the treatment of latent tuberculosis infection with 6 months of isoniazid or 3 months of isoniazid plus rifampicin for migrants from Eastern Europe, South and Central America, Africa, Asia in Spain [33]. The second category of intervention, community education, consisted of small group workshops conducted by the healthcare professionals or staff. They included a small-group discussion on hepatitis B virus testing for Korean and other Asian migrants in the US [23, 27], a walking program and encouragement from older adults in the community or church authority for Chinese migrants in the US [28], the importance of breast or cervical cancer routine screening for Samoan [22], Chinese [26], Hmong [29], and Latin [32] migrants in the US, and the importance of diet and physical activity for Pakistan migrants in Norway [34]. The last group of interventions were peer navigator-related interventions which provided necessary support, follow-up, or referral methods by leaders or lay health workers in the community to help migrants to receive the intervention. Examples included the provision of the information on the importance of breast or cervical cancer and screening program by community staff for the Chinese [26] and Latin [32] migrants in the US, the use of media education and telephone communication provided by lay health workers to provide information about breast cancer screening for Vietnamese migrants in the US [30].

\section{Quality assessment}

Six of 8 RCT studies were rated as a high potential risk of bias due to bias in deviations from intended interventions and bias in outcome measurement. The quality assessment for randomized trials included in this review is shown in Table 4. Quality assessment for quasi-experimental studies were found to be moderate and serious risk of bias for 4 and 1 studies, respectively. Further detail on quality assessment for quasi-experimental studies included in this review is presented in Table 5.

\section{Assessing evidence of inequity based on PROGRESS-Plus}

We found none of the included studies reported the effect of the intervention on reducing inequity according to PROGRESS-Plus. However, all included studies reported the potential difference of the effect of the intervention on outcomes based on PROGRESS-Plus. In addition, six out of 13 studies explicitly discussed the effect of the intervention on equity attributes based on PROGRESS-Plus factors [24, 
Table 4 Quality assessment for randomized controlled trials included in this review

\begin{tabular}{|c|c|c|c|c|c|c|}
\hline \multirow[t]{2}{*}{ Study } & \multicolumn{5}{|l|}{ Domain } & \multirow{2}{*}{$\begin{array}{l}\text { Overall risk } \\
\text { of bias }\end{array}$} \\
\hline & $\begin{array}{l}\text { 1. Randomization } \\
\text { process }\end{array}$ & $\begin{array}{l}\text { 2. Deviation from intended } \\
\text { interventions }\end{array}$ & $\begin{array}{l}\text { 3. Missing } \\
\text { outcome data }\end{array}$ & $\begin{array}{l}\text { 4. Measurement of } \\
\text { outcome }\end{array}$ & $\begin{array}{l}\text { 5.Selection of the } \\
\text { reported results }\end{array}$ & \\
\hline Bastani 2015 & Some concerns $s^{\mathrm{a}, \mathrm{b}}$ & Low risk & Low risk & High risk & Low risk & High risk \\
\hline Braschi 2014 & Some concerns $s^{\mathrm{b}, \mathrm{c}}$ & Low risk & Low risk & Low risk & Low risk & $\begin{array}{l}\text { Some } \\
\text { concerns }\end{array}$ \\
\hline Jandorf 2008 & Some concerns $s^{a, b}$ & Some concerns & Low risk & Low risk & Low risk & $\begin{array}{l}\text { Some } \\
\text { concerns }\end{array}$ \\
\hline $\begin{array}{l}\text { Jimenez-Fuentes } \\
\text { MA } 2013\end{array}$ & High risk & High risk & Some concerns & Some concerns & Some concerns & High risk \\
\hline Mishra, 2007 & High risk & High risk & Low risk & High risk & Low risk & High risk \\
\hline Nguyen, 2009 & Some concerns $s^{a, b}$ & Some concerns & Low risk & High risk & Low risk & High risk \\
\hline $\begin{array}{l}\text { Raberg Kjollesdal } \\
\text { MK } 2011\end{array}$ & Low risk & Some concerns & Some concerns & Low risk & Low risk & High risk \\
\hline Taylor VM 2011 & Some concerns ${ }^{\mathrm{a}, \mathrm{b}}$ & Some concerns & Low risk & High risk & Low risk & High risk \\
\hline
\end{tabular}

${ }^{\mathrm{a}}$ No information was provided about allocation sequence

${ }^{\mathrm{b}} \mathrm{No}$ information was provided about allocation concealment

${ }^{\mathrm{C}} \mathrm{No}$ information was provided about baseline imbalance

26, 27, 30-32]. The determinants included in the studies were: place of residence $(N=2)[22,23]$, language $(N=4)$ [22, 24-26], gender $(N=1)$ [33], religion $(N=2)$ [27, 28], education $(N=5)[22,28,29,33,34]$, socioeconomic status $(N=9)[22,24,26,28-33]$, and age $(N=3)[22,26,30]$. No study examined the difference between occupation and social capital. The frequency of reporting across PROGRESSPlus among included studies is presented in Fig. 3.

\section{Place of residence}

Two studies [22, 23] looked at the association between country of birth and the outcome of an educational program. One RCT [22] focusing on the breast cancer education program among Samoan migrants in the US reported no significant association between country of birth in American Samoa, an unincorporated territory of the United States, compared to other areas of Samoa (OR 1.19, 95\% CI; 0.82-1.74, p 0.365). Another RCT [23] showed the association between country of birth and hepatitis B knowledge score among Asian migrants in the US who participated in classes in English as a second language curriculum which provided hepatitis $B$ virus information. Mean scores were higher among the experimental group than the control group, especially the mean score between groups was significant $(p<0.05)$ for China [3.56 (SD 1.14) vs 2.94 (SD 1.25), p 0.02] and other Asian countries (Afghanistan, Cambodia, Hong Kong, Korea, Taiwan or Vietnam) groups [3.82 (SD 0.95) vs 2.44 (SD 1.46), p 0.002].

\section{Race/ethnicity/culture/ language}

Two RCTs [22, 24] and two quasi-experimental studies $[25,26]$ examined the association between language proficiency and the outcome of the intervention. The two RCTs were conducted in the US. One RCT [22] focusing on a breast cancer educational program among Samoan migrants in the US showed that there was no difference in mammogram receipt between interview languages (Samoan vs English, OR 0.75; 95\% CI; 0.52, 1.06, $p<$ 0.106). Another RCT [24] examined the effect of patient

Table 5 Quality assessment for quasi-experimental studies included in this review

\begin{tabular}{|c|c|c|c|c|c|c|c|c|}
\hline \multirow[t]{2}{*}{ Study } & \multicolumn{7}{|l|}{ Domain } & \multirow{2}{*}{$\begin{array}{l}\text { Overall risk } \\
\text { of bias }\end{array}$} \\
\hline & 1. confounding & $\begin{array}{l}\text { 2. selection of } \\
\text { participants } \\
\text { into the study }\end{array}$ & $\begin{array}{l}\text { 3. classification } \\
\text { of intervention }\end{array}$ & $\begin{array}{l}\text { 4. deviations of } \\
\text { intended } \\
\text { interventions }\end{array}$ & $\begin{array}{l}\text { 5. missing } \\
\text { data }\end{array}$ & $\begin{array}{l}\text { 6. measurement } \\
\text { of outcomes }\end{array}$ & $\begin{array}{l}\text { 7.selection of the } \\
\text { reported results }\end{array}$ & \\
\hline Chiang 2009 & Moderate risk & Moderate Risk & Low risk & Low risk & Low risk & Low risk & Low risk & Moderate Risk \\
\hline Elder 2000 & Moderate risk & Low risk & Low risk & Low risk & Low risk & Low risk & Low risk & Moderate risk \\
\hline Fang 2007 & Moderate risk & Low risk & Low risk & Low Risk & Low risk & Moderate Risk & Low risk & Moderate Risk \\
\hline $\begin{array}{l}\text { Kagawa-Singer, } \\
2009\end{array}$ & Moderate risk & $\mathrm{NI}$ & Low risk & $\mathrm{Nl}$ & Low risk & Serious risk & Low risk & Serious risk \\
\hline Wang X 2010 & Moderate risk & Moderate risk & Low risk & Low risk & Low risk & Moderate risk & Low risk & Moderate risk \\
\hline
\end{tabular}




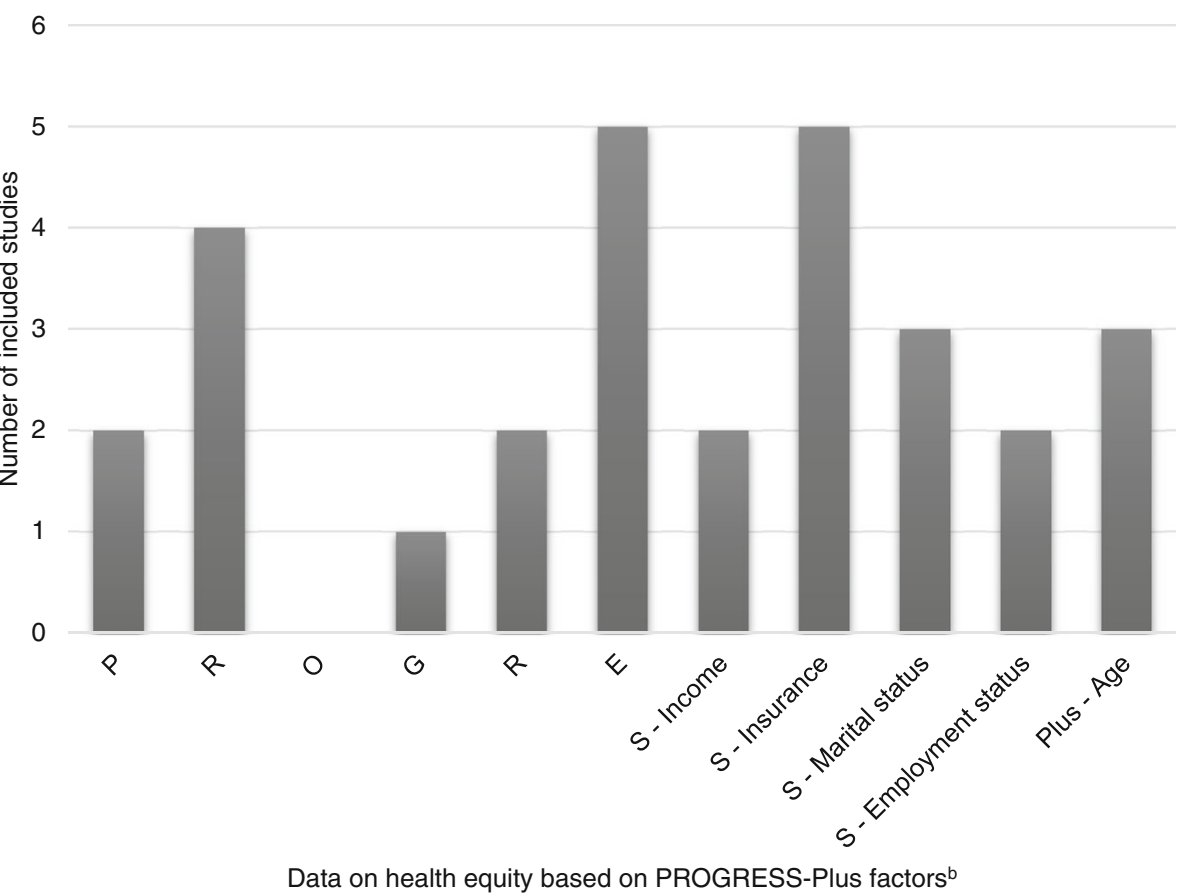

Fig. 3 Studies reported the potential difference of the effect of intervention on outcome-based PROGRESS-Plus ${ }^{\mathrm{a}}$ in this review. ${ }^{\mathrm{a}}$ Data on health equity: PROGRESS-Plus - Place of residence, Race/ethnicity/culture/language, Occupation, Gender/sex, Religion, Education, Socioeconomic status,

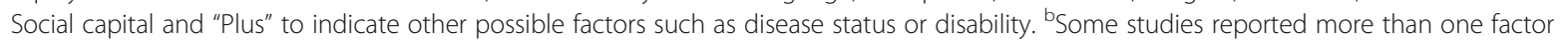

navigation by calling prior to a screening colonoscopy procedure and mailing bowel preparation among Latin migrants. The multiple regression analysis revealed that the language acculturation subscale in Latin migrants was a significant predictor of colonoscopy completion (OR 2.223; 95\% CI; 1.470-3.361, $p<0.001$ ).

One quasi-experimental study [25] assessed the effect of incorporating nutritional behaviour change materials into curricula among Latin migrants in the US. The authors mentioned that nutrition knowledge gain was greater among migrants with medium and high Spanish literacy than those with low literacy. Another study [26] assessed the impact of a community-based intervention that combined cervical cancer education with patient navigation for Chinese migrants in the US and revealed that women with poorer English proficiency were less likely to obtain cervical cancer screening at 12-month follow up compared to those with higher English fluency (OR 0.30; 95\% CI; 0.10-0.89, $p<0.05$ ).

\section{Gender}

We found one RCT [33] examining the difference in non-adherence to preventive chemotherapy of latent tuberculosis infection between males and females among migrant populations (including Eastern Europe,
South and Central America, Africa and Asia) in Spain. Gender was not associated with non-adherence when comparing male to female migrants (OR 1.4; 95\%CI; 0.77-1.69, p 0.49).

\section{Religion}

We found one RCT [27] and one quasi-experimental study [28] assessing the association between religion and the outcome. The RCT [27] was conducted in the US to evaluate effectiveness of a church-based intervention with small group discussion supplemented by materials about hepatitis B screening among Korean migrants to improve Hepatitis B virus testing at 6-month follow up. Statistically significant intervention effects were observed within small (OR 5.3; 95\%CI; 1.7-16.5, p 0.004), medium (OR 6.4; 95\%CI; 2.5-16.3, $p<0.001$ ), and non-Korean town churches (OR 8.6; 95\%CI 3.9-19.4, $p$ $<0.001)$, compared to the control group. A pre-post quasi-experimental study [28] aimed to assess the effect of culturally modified walking with encouragement from older adults in the community among Chinese migrants in the US. The results showed that Taoists or Buddhists spent more time walking than Christians, including Catholics $(p<0.05)$ but no difference in blood pressure was observed across religious groups. 


\section{Education}

Two quasi-experimental studies [28, 29] and three RCTs $[22,33,34]$ looked at the association between education level and health outcomes. The first quasi-experimental study [28] was a culturally modified walking program among Chinese migrants in the US. The study showed that participants with middle school or lower education walked more than those with higher education $(p<$ $0.05)$. However, it was found that the decrease in systolic blood pressure was much larger in those with higher education than those with lower education $(p<0.05)$ at post-test in the intervention group. The second quasi-experimental study [29] assessed the effect of an education program about the importance and steps of breast cancer screening among Hmong migrants in the US. The subgroup analysis revealed that breast self-examination screening increased in participants with no schooling in the intervention group compared with the control group (OR 4.32; 95\%CI; 1.05-17.71, $p<$ $0.05)$. However, no difference in clinical breast examination and mammography receipt between groups was detected. One RCT [22] provided a breast cancer educational program among Samoan migrants in the US. The result showed there was no difference in mammogram receipt across education level when comparing women with more than 12 years of education (OR 1.55; 95\%CI; $0.98-2.45$, p 0.063) and women with 9-12 years of education (OR 1.19, 95\%CI; 0.88-1.60, p 0.259) to women with equal or less than 8 years of education. Another RCT [34] examined the effect of a group session using culturally adapted materials with a discussion panel focusing on the importance of diet and physical activity for blood glucose regulation among Pakistan migrants in Norway. The results revealed that changes in the perceptions in the intervention group were not significantly related to age, number of years in Norway, years of education or command of the Norwegian language, with the exception that those with higher education had changed their perception of legumes as good for the body (OR 1.13, p 0.01). The third RCT [33] reported the effect of a treatment of latent tuberculosis infection among migrant populations in Spain. The univariate analysis indicated that illiteracy was associated with non-adherence to drug treatment (OR 1.73; 95\%CI; 1.04-2.88, p 0.02).

\section{Socio-economic status Income}

One RCT [24] examined the effect of patient navigation intervention among Latin migrants in the US. The study found that those with higher income levels had greater uptake of preventive service. In particular, an annual income above $\$ 10,000$ was an independent predictor of the completion of colonoscopy screening from patient navigation program compared to those with income below $\$ 10,000$ (OR 1.97, 95\%CI; 1.09-3.56, p 0.026).

\section{Insurance status}

Three RCTs [22, 24, 30] and two quasi-experimental studies $[26,31]$ evaluated the association between insurance status and their health outcomes. The first RCT [24] showed that insurance status was not associated with completion of colonoscopy screening (OR 2.54; 95\%CI; 0.82-3.68, p 0.11) when comparing the private/self-pay insurance group to the Medicare/Medicaid scheme group among Latin migrants in the US who received the intervention. The second RCT [22] mentioned that there was no difference in mammogram receipt between insurance status (OR 1.21; 95\% CI; 0.92-1.97, p 0.125) in the intervention group among Samoan migrants in the US who attended the breast cancer educational program. However, the third RCT [30] conducted a program by using lay health workers with media education about cervical cancer screening among Vietnamese migrants in the US. The study revealed that participants with health insurance increased mammogram receipt within 2 years compared to the group with no insurance (OR 2.84; 95\%CI; 1.73-4.69) Moreover, two quasi-experimental studies $[26,31]$ revealed that insurance status was significantly associated with completion of the screening program. One quasi-experimental study [31] provided small group educational sessions focusing on cervical cancer and the benefits of screening and early detection among Korean migrants in the US. The multiple logistic regression demonstrated that insurance status was significantly associated with cervical cancer screening uptake (OR 9.53; 95\% CI; 1.30-69.66, p 0.03). Another study [26] evaluated a community-based intervention which combined cervical cancer education with patient navigation to increase the receipt of a screening program among Chinese migrants in the US. Women without health insurance were less likely to obtain the screening program, compared to women with health insurance (OR 0.15; 95\%CI; 0.02-0.96, $p<0.05$ ).

\section{Marital status}

One quasi-experimental study [31] examined the effect of a culturally modified walking program among Korean migrants in the US and revealed that marital status was not associated with screening uptake for cervical cancer (OR 0.78; 95\%CI; 0.17-3.49, p 0.74). However, two RCTs [22, 32] showed that women who were married were significantly more adherent to screening uptake for breast cancer. One RCT [32] provided an educational session of cervical and breast cancer among Latin migrants in the US. This study demonstrated that women who were married or living with partners were significantly more likely to have had a clinical breast examination (OR 2.0; 95\%CI; 1.1-3.7, p 0.03). In addition, another RCT [22] assessed a breast cancer education program among Samoan in the US. 
Mammogram receipt with current married status was significantly higher than those who were single (OR 1.31; 95\%CI; 1.01-1.70, p 0.041).

\section{Employment status}

Two RCTs [22, 33] showed that unemployment status might lead to poor outcomes. One RCT [33] reported that unemployment status was associated with non-adherence to drug treatment for latent tuberculosis among migrants in Spain (OR 1.91; 95\%CI; 1.01-2.15, p 0.03). Another RCT [22] provided a breast cancer educational program and demonstrated that those who were currently employed increased self-reported receipt of mammogram among Samoan migrants in the US, compared to those who were unemployed (OR 1.48; 95\%CI; 1.15-1.13, p 0.005).

\section{Others}

In addition to PROGRESS as determinants of health intervention effects, three studies $[22,26,30]$ examined the age of migrants as 'plus' or other determinants of health equity. Two RCTs [22, 30] and one quasi-experimental study [26] examined the association between age and outcomes.

One RCT [22] evaluated the effect of breast cancer education program among Samoan migrants in the US. The study found no difference in mammogram receipt among age groups when comparing participants aged $42-49$ years (OR 1.21; 95\%CI; 0.82-1.80, p 0.337) with those aged 5064 years (OR 1.29; 95\%CI; 0.90-1.86, p 0.171) to participants aged 65 years or older, respectively. Another RCT [30] examined the effect of lay health workers with media education for breast cancer screening among Vietnamese migrants in the US. The multivariate analysis demonstrated that the participants aged 40-49 years had fewer mammograms within 2 years, compared to those aged 50-64 years (OR 0.51, 95\%CI; 0.30-0.87). In contrast, the participants aged 65 years or older had fewer clinical breast examinations within 2 years compared to those aged $50-64$ years (OR 0.51, 95\%CI; 0.31-0.81).

One quasi-experimental study [26] showed that the outcomes in the 12-month interval following a communitybased program among Chinese migrants in the US, cervical screening rate were significantly higher in the intervention group (70\%) compared to the control group (11.1\%). Hierarchical logistic regression analysis indicated that screening behavior was associated with older age (OR 1.08, 95\% CI; $1.01-1.15, p<0.05)$.

\section{Discussion}

We systematically identified experimental studies that evaluated the effects of interventions on health outcomes of migrant populations and assessed whether equity was addressed in the published literature based on PROGRESSPlus factors. None of the included studies examined the effect of health interventions on reducing inequity amongst migrant populations. However, some studies reported the potential difference of the effect of the intervention on outcomes based on PROGRESS-Plus. Our findings suggest for the need to develop interventions to improve health outcomes of migrants and incorporate the equity attributes as part of outcome measurement, to support the goal to achieve SDG on the reduction of inequalities $[1,5]$.

Our results are consistent with the findings from previously published reviews on interventions related to vaccination [36] and health care models among migrant populations [37]. Both reviews [36, 37] similarly mentioned that none of the included studies reported the effectiveness or measured the impact of interventions on health inequity in the populations. In terms of inclusion of equity attributes in the studies, only $3.41 \%$ in our review (13 out of 381 experimental studies) reported variation in the outcomes by equity attributes. Less than half of these discussed equity issues in their discussion and conclusions. This is similar to what was reported in a previous review [36] which reported no increasing trend of consideration of equity attributes in studies over time. These findings highlight a lack of research interest in measuring the effects of interventions in relation to equity attributes among migrant populations. Since addressing equity as part of outcome measurement is an important part of meeting the SDGs; more research on health interventions for migrants incorporating equity attributes based on PROGRESS-Plus factors is strongly encouraged in the future.

The review approach adopted in our review is somewhat different from those in previous reviews. Since we wanted to look at how primary studies on health interventions for migrants have captured equity attributes, we needed to determine a feasible approach that would enable us to identify primary studies. Given the extremely large number of individual studies identified from searching, we chose to use a two-stage systematic review approach. The unit of analysis in our review was at the individual study, while at the review level for previous reviews. We extracted information from primary studies to evaluate the effect of health interventions based on the equity attributes while the previous study [36] performed an overview of systematic reviews. We also included only experimental studies while the previous study [36] included both experimental and observational studies and another study [37] included qualitative research and policy documents.

Several limitations inherent in our review deserve discussion. First, all included studies were conducted in high-income countries including the US, Spain, and Norway. Therefore, our findings of the lack of minimal measurement of equity attributes might have limited generalizability to those studies conducted in low and middle-income countries. Second, six out of 8 included RCTs potentially had a high risk of methodological bias. 
There remains a need for further improvement of the methodology used in studies assessing the effect of interventions among migrant populations.

\section{Conclusion}

This systematic review has critically highlighted the current health intervention studies targeted at migrants. A paucity of such studies indicates that equity has not been receiving attention and greater attention to equity-focused outcome assessment is needed. To reduce health inequities among these populations, the framework which includes equity attributes based PROGRESS-Plus factors should be incorporated into future implementation research.

\section{Additional file}

Additional file 1: Table S1. Search strategy (from inception until June 2017). (DOCX $28 \mathrm{~kb}$ )

\section{Abbreviations}

OR: Odds ratio; PROGRESS-Plus : Place of residence, race/ethnicity, occupation, gender, religion, education, socio-economic status, social capital and others; RCT: Randomized controlled trial; SD: Standard deviation; SDGs : Sustainable Development Goals; UN: The United Nations; US: The United States; WHO: World Health Organization

\section{Acknowledgements}

Not applicable.

\section{Funding}

The authors did not receive any funding for this work.

\section{Availability of data and materials}

Authors can confirm that all relevant data are included in the article and/or its supplementary information files.

\section{Authors' contributions}

JY conducted the literature search, performed the screening and information extraction from the included studies. MB verified the extracted data. NC and $\mathrm{KT}$ independently performed the eligibility and quality assessment of the full texts of articles which passing the second stage screening. All authors read and approved the final manuscript.

\section{Ethics approval and consent to participate}

Not applicable.

\section{Consent for publication}

Not applicable

\section{Competing interests}

The authors declare that they have no competing interests.

\section{Publisher's Note}

Springer Nature remains neutral with regard to jurisdictional claims in published maps and institutional affiliations.

\section{Author details}

'Department of Pharmaceutical Care, Faculty of Pharmacy, Chiang Mai University, Chiang Mai, Thailand. ${ }^{2}$ Evidence for Policy and Practice Information and Coordinating Centre (EPPI-Centre), UCL Institute of Education, University College London, London, UK. ${ }^{3}$ Ottawa Hospital Research Institute, The Ottawa Hospital, Ottawa, Ontario, Canada. ${ }^{4}$ School of Epidemiology and Public Health, Faculty of Medicine, University of Ottawa, Ottawa, Ontario, Canada. ${ }^{5}$ Institute of Clinical and Evaluative Sciences, ICES uOttawa, Ottawa, Ontario, Canada. ${ }^{6}$ Bruyere Research Institute, University of
Ottawa, Ottawa, Ontario, Canada. ${ }^{7}$ Department of Medicine, Faculty of Medicine, University of Ottawa, Ottawa, Ontario, Canada. ${ }^{8}$ Clinical Epidemiology Program, Ottawa Hospital Research Institute, Ottawa, Ontario, Canada. 'WHO Collaborating Centre for Knowledge Translation and Health Technology Assessment in Health Equity, Bruyère Research Institute, Ottawa, Ontario, Canada. ${ }^{10}$ School of Pharmacy, Monash University Malaysia, Jalan Lagoon Selatan, 46150 Bandar Sunway, Selangor, Malaysia. " Center of Pharmaceutical Outcomes Research (CPOR), Department of Pharmacy Practice, Faculty of Pharmaceutical Sciences, Naresuan University, Phitsanulok, Thailand. ${ }^{12}$ School of Pharmacy, University of Wisconsin, Madison, USA.

${ }^{13}$ Asian Centre for Evidence Synthesis in Population, Implementation and Clinical Outcomes (PICO), Health and Well-being Cluster, Global Asia in the 21st Century (GA21) Platform, Monash University Malaysia, Bandar Sunway, Selangor, Malaysia. ${ }^{14}$ Department of Pharmacotherapy, College of Pharmacy, University of Utah, Salt Lake City, Utah, USA.

Received: 7 August 2018 Accepted: 22 April 2019

Published online: 06 May 2019

\section{References}

1. The United Nations. Goal 10: reduce inequality within and among countries Available from: http://www.un.org/sustainabledevelopment/inequality.

2. International Organization for Migration. World migration report 2018.

3. Migration Policy Institute tabulation of data from the United Nations, Department of Economic and Social Affairs. Trends in international migrant stock: migrants by destination and origin 2015 Available from: http://www. un.org/en/development/desa/population/migration/data/estimates2/ estimates $15 . \mathrm{shtml}$.

4. World Health Organization. Constitution of the World Health Organization. Geneva: World Health Organization; 1948.

5. World Health Organization. World health statistics 2017: monitoring health for the SDGs, sustainable development goals. Geneva: World Health Organization; 2017.

6. Diaz E, Ortiz-Barreda G, Ben-Shlomo Y, Holdsworth M, Salami B, Rammohan A, et al. Interventions to improve immigrant health. A scoping review. Eur J Pub Health. 2017;27(3):433-9.

7. Fernandez-Gutierrez M, Bas-Sarmiento P, Albar-Marin MJ, Paloma-Castro O, Romero-Sanchez JM. Health literacy interventions for immigrant populations: a systematic review. Int Nurs Rev. 2017 Apr 27. [Epub ahead of print].

8. Aung E, Blondell SJ, Durham J. Interventions for increasing HIV testing uptake in migrants: a systematic review of evidence. AIDS Behav. 2017; 21(10):2844-59.

9. Genoff MC, Zaballa A, Gany F, Gonzalez J, Ramirez J, Jewell ST, et al. Navigating language barriers: a systematic review of patient navigators' impact on cancer screening for limited english proficient patients. J Gen Intern Med. 2016;31(4):426-34.

10. Heo HH, Braun KL. Culturally tailored interventions of chronic disease targeting Korean Americans: a systematic review. Ethn Health. 2014;19(1):64-85.

11. Joo JY. Effectiveness of culturally tailored diabetes interventions for asian immigrants to the United States. Diabetes Educ. 2014;40(5):605-15.

12. Martin CA, Gowda U, Smith BJ, Renzaho AM. Systematic review of the effect of lifestyle interventions on the components of the metabolic syndrome in south asian migrants. J Immigr Minor Health. 2016 Oct 21. [Epub ahead of print].

13. Renzaho AM, Mellor D, Boulton K, Swinburn B. Effectiveness of prevention programmes for obesity and chronic diseases among immigrants to developed countries - a systematic review. Public Health Nutr. 2010;13(3):438-50.

14. Tovar A, Renzaho AMN, Guerrero AD, Mena N, Ayala GX. A systematic review of obesity prevention intervention studies among immigrant populations in the US. Curr Obes Rep. 2014;3(2):206-22.

15. Welch V, Petticrew M, Tugwell P, Moher D, O'Neill J, Waters E, et al. PRISMAequity 2012 extension: reporting guidelines for systematic reviews with a focus on health equity. PLoS Med. 2012;9(10):e1001333.

16. International organization of migration. Key migration terms 2016 Available from: http://www.iom.int/key-migration-terms.

17. O'Neill J, Tabish H, Welch V, Petticrew M, Pottie K, Clarke M, et al. Applying an equity lens to interventions: using PROGRESS ensures consideration of socially stratifying factors to illuminate inequities in health. J Clin Epidemiol. 2014;67:56-64. 
18. Higgins JP, Sterne JA, Savović J, Page MJ, Hróbjartsson A, Boutron I, et al. A revised tool for assessing risk of bias in randomized trials. Cochrane Database Syst Rev. 2016;10(suppl 1):29-31.

19. Sterne JA, Hernán MA, Reeves BC, Savović J, Berkman ND, Henry MV. ROBINS-l: a tool for assessing risk of bias in non-randomised studies of interventions. BMJ. 2016;355:14919.

20. Lucas PJ, Baird J, Arai L, Law C, HM R. Worked examples of alternative methods for the synthesis of qualitative and quantitative research in systematic reviews. BMC Med Res Methodol. 2007;7(4).

21. Barnett-Page ETJ. Methods for the synthesis of qualitative research: a critical review. BMC Med Res Methodol. 2009:9(1):59.

22. Mishra SI, Bastani R, Crespi CM, Chang LC, Luce PH, Baquet CR. Results of a randomized trial to increase mammogram usage among samoan women. Cancer Epidemiol Biomark Prev. 2007;16(12):2594-604.

23. Taylor VM, Gregory Hislop T, Bajdik C, Teh C, Lam W, Acorda E, et al. Hepatitis B ESL education for asian immigrants. J Community Health. 2011; 36(1):35-41.

24. Braschi CD, Sly JR, Singh S, Villagra C, Jandorf L. Increasing colonoscopy screening for Latino Americans through a patient navigation model: a randomized clinical trial. J Immigr Minor Health. 2014;16(5):934-40.

25. Elder JP, Candelaria JI, Woodruff SI, Criqui MH, Talavera GA, Rupp JW. Results of language for health: cardiovascular disease nutrition education for latino english-as-a-second-language students. Health Educ Behav. 2000;27(1):50-63.

26. Wang X, Fang C, Tan Y, Liu A, Ma GX. Evidence-based intervention to reduce access barriers to cervical cancer screening among underserved chinese american women. J Women's Health. 2010;19(3):463-9.

27. Bastani R, Glenn BA, Maxwell AE, Jo AM, Herrmann AK, Crespi CM, et al. Cluster-randomized trial to increase hepatitis B testing among Koreans in Los Angeles. Cancer Epidemiol Biomark Prev. 2015;24(9):1341-9.

28. Chiang CY, Sun FK. The effects of a walking program on older Chinese American immigrants with hypertension: a pretest and posttest quasiexperimental design. Public Health Nurs. 2009;26(3):240-8.

29. Kagawa-Singer M, Tanjasiri SP, Valdez A, Yu H, Foo MA. Outcomes of a breast health project for Hmong women and men in California. Am J Public Health. 2009;99(Suppl 2):S467-73.

30. Nguyen TT, Le G, Nguyen T, Le K, Lai K, Gildengorin G, et al. Breast cancer screening among vietnamese americans: a randomized controlled trial of lay health worker outreach. Am J Prev Med. 2009;37(4):306-13.

31. Fang CY, Ma GX, Tan Y, Chi N. A multifaceted intervention to increase cervical cancer screening among underserved Korean women. Cancer Epidemiol Biomark Prev. 2007;16(6):1298-302.

32. Jandorf L, Bursac Z, Pulley L, Trevino M, Castillo A, Erwin DO. Breast and cervical cancer screening among Latinas attending culturally specific educational programs. Prog Community Health Partnersh. 2008;2(3):195-204.

33. Jimenez-Fuentes MA, de Souza-Galvao ML, Mila Auge C, Solsona Peiro J, AltetGomez MN. Rifampicin plus isoniazid for the prevention of tuberculosis in an immigrant population. Int J Tuberc Lung Dis. 2013;17(3):326-32.

34. Råberg Kiøllesdal MK, Hjellset VT, Bjørge B, Holmboe-Ottesen G, Wandel M. Food perceptions in terms of health among Norwegian-Pakistani women participating in a culturally adapted intervention. Int J Public Health. 2011; 56(5):475-83.

35. Han HR, Kim J, Lee JE, Hedlin HK, Song H, Song Y, et al. Interventions that increase use of pap tests among ethnic minority women: a meta-analysis. Psycho-Oncology. 2011;20(4):341-51.

36. Bosch-Capblanch X, Zuske MK, Auer C. Research on subgroups is not research on equity attributes: evidence from an overview of systematic reviews on vaccination. Int J Equity Health. 2017;16(95):1-10.

37. Batista R, Pottie K, Bouchard L, Ng E, Tanuseputro P, Tugwell P. Primary health care models addressing health equity for immigrants: a systematic scoping review. J Immigr Minor Health. 2018;20:214-30.

Ready to submit your research? Choose BMC and benefit from:

- fast, convenient online submission

- thorough peer review by experienced researchers in your field

- rapid publication on acceptance

- support for research data, including large and complex data types

- gold Open Access which fosters wider collaboration and increased citations

- maximum visibility for your research: over $100 \mathrm{M}$ website views per year

At BMC, research is always in progress.

Learn more biomedcentral.com/submissions 